\title{
Development and Evaluation of a 193nm Immersion Generation-Three Fluid Candidates
}

\author{
${ }^{1}$ Paul A. Zimmerman, ${ }^{1}$ Jeff Byers, ${ }^{1}$ Bryan Rice, ${ }^{2}$ Christopher K. Ober, ${ }^{2}$ Emmanuel P. Giannelis, ${ }^{2}$ Robert \\ Rodriguez, ${ }^{2}$ Dongyan Wang, ${ }^{3}$ Naphtali O'Connor, ${ }^{3}$ Xuegong Lei, ${ }^{3}$ Nicholas J. Turro, ${ }^{4}$ Vladimir Liberman \\ ${ }^{4}$ Stephen Palmacci,; ${ }^{4}$ Mordechai Rothschild, ${ }^{5}$ Neal Lafferty, ${ }^{5}$ Bruce, W. Smith \\ ${ }^{1}$ SEMATECH, ${ }^{2}$ Cornell University, ${ }^{3}$ Columbia University, ${ }^{4}$ MIT-LL, ${ }^{5}$ Rochester Institute of Technology \\ paul.zimmerman@intel.com
}

\begin{abstract}
The need to extend 193nm immersion lithography necessitates the development of a third generation (Gen-3) of high refractive index (RI) fluids that will enable approximately 1.7 numerical aperture (NA) imaging. A multipronged approach was taken to develop these materials. One approach investigated the highest-index organic thus far discovered. The second approach used a very high refractive index nanoparticle to make a nanocomposite fluid.

This report will describe the chemistry of the best Gen-3 fluid candidates and the systematic approach to their identification and synthesis. Images obtained with the Gen-3 fluid candidates will also be presented for a NA $\geq 1.7$
\end{abstract}

Keywords: Immersion lithography, refractive index, immersion fluid, nanoparticles

\section{INTRODUCTION}

The introduction of $193 \mathrm{~nm}$ lithography using water has allowed optical lithography to be extended beyond the $45 \mathrm{~nm}$ half-pitch node. Water, nearly the perfect fluid (reasonably inert, relatively high index compared to air, inexpensive, non-toxic, transparent, low viscosity, etc.), was easily introduced into the semiconductor manufacturing environment. Introducing any other liquid as an immersion fluid will require expensive new materials and significant equipment modifications. Significant effort was originally put into finding a higher refractive index (RI) aqueous fluid. ${ }^{1}$ These results yielded fluids with indices up to about $1.54 .^{1}$

Major materials suppliers took the approach of investigating organic-based fluids ${ }^{2-4}$. All of these fluids had an index around 1.64 at $193 \mathrm{~nm}$. While the initial transparency for these fluids in pure form is good, in every case the fluids degrade rapidly under exposure to atmosphere. These organics require an in-line remediation to maintain their transparency for reasonable timeframes. ${ }^{6,7}$ It has also been shown that all organic fluids are likely to eventually degrade during $193 \mathrm{~nm}$ exposure. ${ }^{7}$ The degradation of the fluid is of great concern because lens deposits that form during exposure may cause irreversible damage. ${ }^{8}$ It has been several years since the introduction of a decalin like immersion fluid, and continual improvement has reduced the absorbance to $\sim 0.02 / \mathrm{cm}$. However, in the past two years no other organics have been shown to be viable even at an absorbance of $0.25 / \mathrm{cm}$. Many possible target materials have been suggested, but in each case increases in the index have been accompanied by unacceptable levels of absorbance. ${ }^{9-11}$ Part of this report illustrates the difficulty we faced in developing an acceptable transparent organic immersion fluid with an index greater that 1.7. Even more challenging is the industry goal of reaching a RI $\geq 1.80$ necessary for $\sim 1.7$ numerical aperture imaging. ${ }^{12-14}$ This number was obtained through extensive simulation, but little thought was given to the actual chemistry that would be required to achieve the requisite RI.

\subsection{Chemistry and Refractive index}

Creating a target number for any process is often the easiest part of a project. If we examine how we can affect the refractive index of, for example, non-polar or semi-polar organic fluids, we can look at the relationship shown in equation 1 below. Here, $\eta$ is the absolute index of refraction, $K_{d}$ is the relative permittivity (dielectric constant), and $\mathrm{K}_{\mathrm{m}}$ is the relative permeability. It is a good assumption that for all organic species that $\mathrm{K}_{\mathrm{m}}$ will be approximately one, leaving $\mathrm{K}_{\mathrm{d}}$ the relative permittivity (dielectric constant) as the only way to dramatically affect the refractive index. Although this relationship is an oversimplification and does not describe the behavior of a material versus wavelength,

Advances in Resist Materials and Processing Technology XXV, edited by Clifford L. Henderson, Proc. of SPIE Vol. 6923, 69230A, (2008) · 0277-786X/08/\$18 - doi: 10.1117/12.772887 
the latter can be extracted with the Kramers-Kronig relation. ${ }^{15}$ Still equation 1 indicates that changing the dielectric constant should result in an increase in the RI.

$$
\eta \quad=\sqrt{\mathbf{K}_{\mathbf{d}} \mathbf{K}_{\mathbf{m}}} \text { (Equation 1) }
$$

Increasing the dielectric constant (i.e., the polarizability) of a chemical system can be accomplished through various means. Some of these are summarized in Table 1. For example, the trend is clear with respect to adding ever more polarizable groups. The addition of the larger halides results in a considerable increase in RI compared to the corresponding normal hydrocarbon. When adding $\mathrm{F}$, the index decreases relative to the hydrocarbon. This is because of the lack of polarizability of the $\mathrm{F}$ atom. If you take any alkyl halide and add additional methylene groups, as shown in the table, RI increases significantly, for example, going from methyl iodide to t-butyliodide. Clearly, adding any $\mathrm{C}_{\mathrm{n}} \mathrm{H}_{2 \mathrm{n}}$ reduces the RI dramatically. Also shown in the table is the impact to RI of adding double or triple bounds. Further, adding rings and aromaticity significantly increases the RI compared to analogous n-chain hydrocarbons. However, at $193 \mathrm{~nm}$ most of these moieties cannot be used due to high absorbance or reactivity.

For the last two years, the approach has been to search for pure hydrocarbon compounds beyond decalin that have a similar absorbance but higher RI. Figure 1 illustrates a trend that was identified for pure hydrocarbon species showing that RI is more or less proportional to the material density. It was easy to find the densest hydrocarbons, purify them, and then measure the RI and absorbance. This procedure has been detailed elsewhere. ${ }^{10}$ However, it has been highlighted by several groups that increasing the size of the organic compound while raising the RI also increases the absorbance to unacceptable levels. ${ }^{10,11,16}$ Figure 2 shows an example of increased RI when going from decalin to perhydrofluorene and perhydropyrene. By the time you get to perhydropyrene, however, the absorbance becomes greater than $2.5 / \mathrm{cm}$, which is far too high to be useful for $193 \mathrm{~nm}$ immersion lithography. In the search for an exception that might somehow challenge the observed trend, methylcubane was evaluated. With a density greater than water, it offered the best opportunity to achieve a RI of 1.8 with a pure organic fluid. Density functional theory calculations for cubane systems showed the possibility of a dip in the absorbance between two transitions that may occur, ${ }^{17}$ but while the RI was high, the evaluation of methylcubane also showed no trough in the absorbance spectrum and an ultraviolet (UV) cutoff between 230-240nm, making methylcubane unusable as a 193nm immersion fluid.

The current thinking is that a pure hydrocarbon organic immersion fluid is not fundamentally possible. However, before the organic approach was completely abandoned, the use of multiple types of heteroatoms was investigated (Table 1). Clearly the introduction of heteroatoms (i.e. S, N, P etc) increases polarizability. Table 1 shows the change in RI when adding the different Group VII elements, the halides. Clearly, the addition of the more polarizable halides dramatically increases the RI. Tables 2, 3, and 4 show examples of molecular species from Group IV, Group V, and Group VI, respectively

Table 1 showing some trends of the RI with the introduction of constituents to impact polarizability

\begin{tabular}{c|c}
\hline Compound & $\mathrm{RI}(589 \mathrm{~nm})$ \\
\hline $\mathrm{CH}_{\mathbf{3}}\left(\mathrm{CH}_{\mathbf{2}}\right)_{\mathbf{4}} \mathbf{F}$ & 1.360 \\
\hline $\mathrm{CH}_{\mathbf{3}}\left(\mathrm{CH}_{\mathbf{2}}\right)_{\mathbf{4}} \mathrm{Cl}$ & 1.412 \\
\hline $\mathrm{CH}_{\mathbf{3}}\left(\mathrm{CH}_{\mathbf{2}}\right)_{\mathbf{4}} \mathbf{B r}$ & 1.444 \\
\hline $\mathrm{CH}_{\mathbf{3}}\left(\mathrm{CH}_{\mathbf{2}}\right)_{\mathbf{4}} \mathrm{I}$ & 1.495 \\
\hline $\mathrm{CH}_{\mathbf{3}} \mathrm{I}$ & 1.531 \\
\hline$\left(\mathrm{CH}_{\mathbf{3}}\right) \mathbf{C I}$ & 1.491 \\
\hline & \\
\hline n-Pentane & 1.358 \\
\hline 1-Pentene & 1.372 \\
\hline 1-Pentyne & 1.385 \\
\hline & \\
\hline n-C6H14 & 1.375 \\
\hline C6H12 & 1.426 \\
\hline C6H6 & 1.501 \\
\hline
\end{tabular}




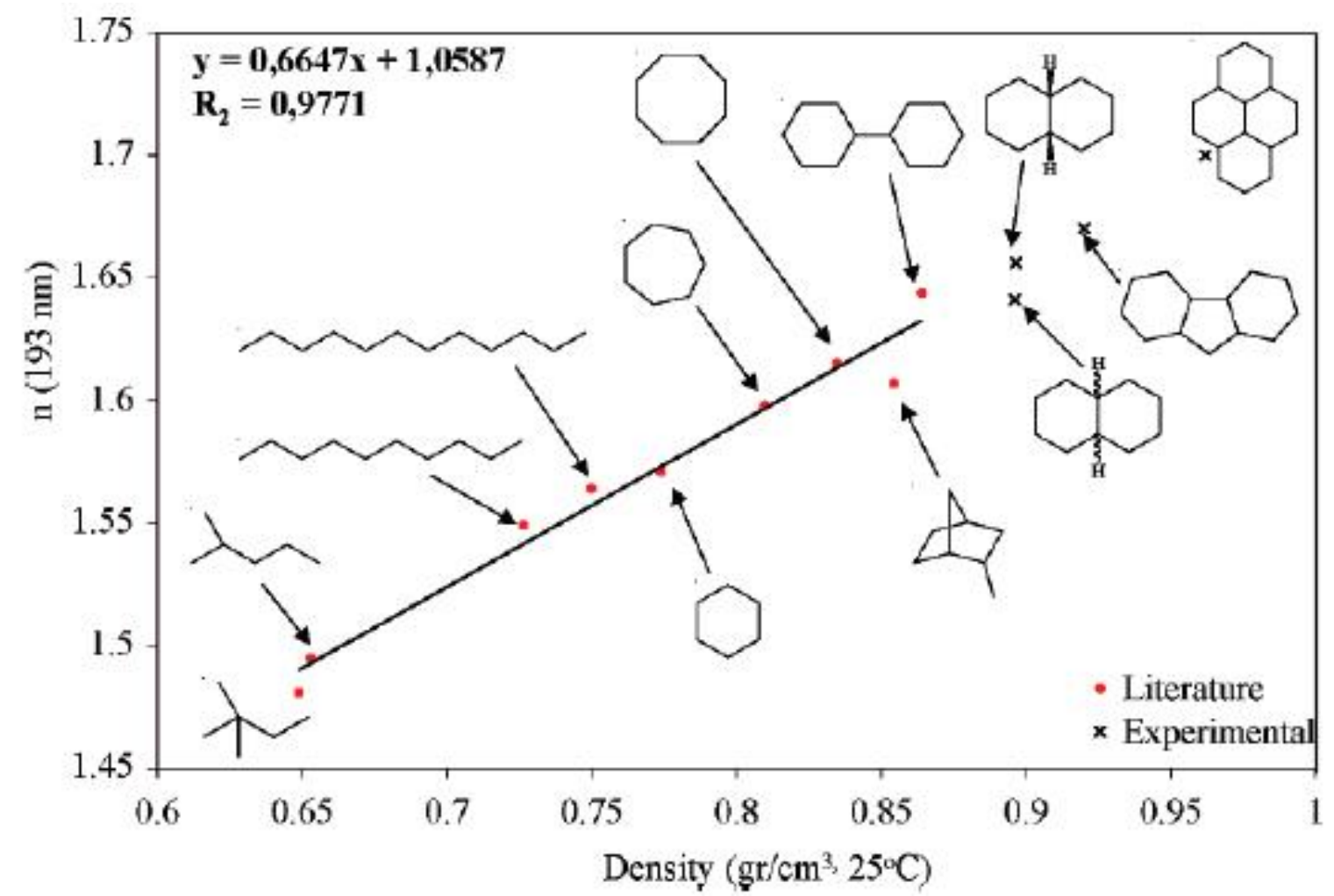

rigure I KI vs. density trend tor saturated nyarocarbons

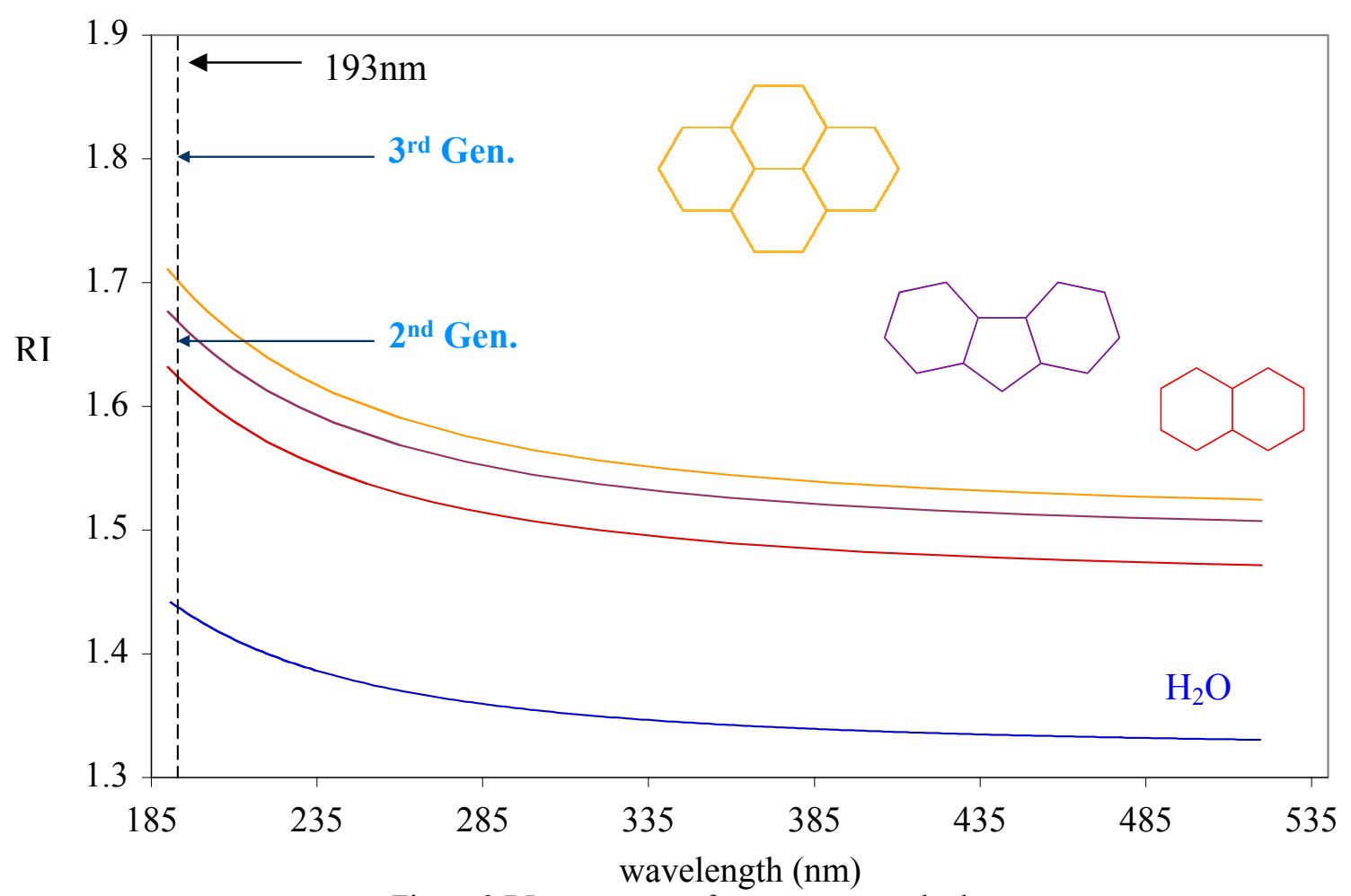

Figure 2 RI vs. structure from water to perhydropyrene 
During the high index project many heteroatom-containing systems were explored, but it rapidly became clear that the index could be only marginally improved by introducing such atoms; in each case, other material properties (i.e., transparency, reactivity, etc.) significantly degraded. For example, substituting nitrogen and/or phosphorus for a carbon can increase the index. When we take it to the extreme, as shown for the compound in Figure 3, we get a higher RI than when a pure hydrocarbon is used. The RI of the compound shown in Figure 3 is 1.517 at $193 \mathrm{~nm}$. The material, however, is a phosphazene and has a $\mathrm{K}_{\mathrm{b}}$ of about 28, which makes it far too basic to be useful in an operational system. Sulfur in the Group VI elements was also examined. The results have been detailed elsewhere. ${ }^{18}$ However, they were unambiguous in that the introduction of sulfur into hydrocarbons does not provide a viable path to produce a high-index immersion fluid.

Table 2 RI changes as more polarizable Group IV elements are substituted for carbon

\begin{tabular}{|c|c|}
\hline Compound & $\mathrm{RI}(589 \mathrm{~nm})$ \\
\hline Tetraethylmethane & 1.418 \\
\hline Tetraethylsilane & 1.427 \\
\hline Tetraethylgermane & 1.443 \\
\hline Tetraethylstannane & 1.473 \\
\hline
\end{tabular}

Table 3 RI changes as more polarizable Group V elements are substituted for nitrogen

\begin{tabular}{|c|c|}
\hline Compound & $\mathrm{RI}(589 \mathrm{~nm})$ \\
\hline Triethylamine & 1.401 \\
\hline Triethylphosphine & 1.456 \\
\hline Triethylarsine & $1.47 ?$ \\
\hline
\end{tabular}

Table 4 RI changes as more polarizable Group VI elements are substituted for oxygen

\begin{tabular}{|c|c|}
\hline Compound & $\mathrm{RI}(589 \mathrm{~nm})$ \\
\hline Diphenyl ether & 1.579 \\
\hline Diphenyl sulfide & 1.632 \\
\hline Diphenyl sellinide & 1.645 \\
\hline Diphenyl telluride & 1.647 \\
\hline
\end{tabular}

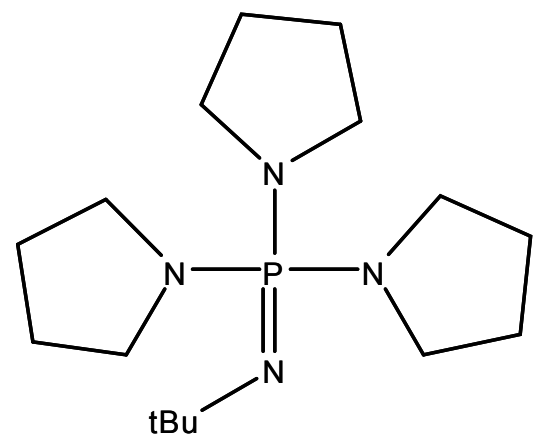

Figure 3 tert-Butyliminotri(pyrrolidino)phosphorane examined as a possible immersion fluid.

If it is possible to accept the data presented thus far, it is not unreasonable to say that the use of a pure organic Gen-3 immersion fluid with an index approaching 1.80 is extremely unlikely. In this case, we must turn our efforts to alternatives for pure organic fluid systems: the introduction of highly polarizable transparent inorganic species in the form of nanoparticles. 


\subsection{Nanoparticles}

The first step in evaluating the viability of a nanoparticle approach for a high-index immersion fluid is to select a material that will significantly increase the RI of the nanocomposite solution. Figure 4 shows the refractive index vs. wavelength for a material that has an index of about 2.90 at $193 \mathrm{~nm}$. Results are shown for film thicknesses of 2, 4, and $6 \mathrm{~nm}$. The thickness of these films is consistent with the thickness range of the nanoparticles being investigated. In evaluating this type of fluid, it is important to consider both fundamental and practical aspects. Among the fundamental considerations are absorbance and scattering induced by the nanoparticles. Practical considerations include nanoparticle synthesis, loading capacity, and possible increase in process-induced defects All of these are being evaluated.

Additionally, two types of nanocomposite solutions, aqueous and organic, are being developed. The aqueous-based system will be designed to compete with Gen-2 fluids with a RI around 1.65 at $193 \mathrm{~nm}$. Of course, this aqueous Gen-2 fluid would be extremely attractive because of its higher stability under exposure conditions compared to the observed degradation with the organic fluids. ${ }^{7,8}$ The material chosen for the nanoparticles has a nearly unique interaction with water. Figure 5 shows the interaction between water and metal oxide. Note that the metal is extremely electropositive while oxygen is quite electronegative, making the system extremely polarizable. Additionally, it is believed that water can coordinate with the metal oxide as shown in Figure 5. Note that there are not only hydrogen bonds but also a Van der Waal's interaction between the oxygen on the water and the metal in the metal oxide. In fact, it is known that very high temperatures $\left(>350^{\circ} \mathrm{C}\right.$ for several hours) are needed to completely remove water from the system. For our application in photolithography, this inability to dehydrate under ambient conditions may help remove the nanoparticles during the development step thereby reducing the chances for defectivity.

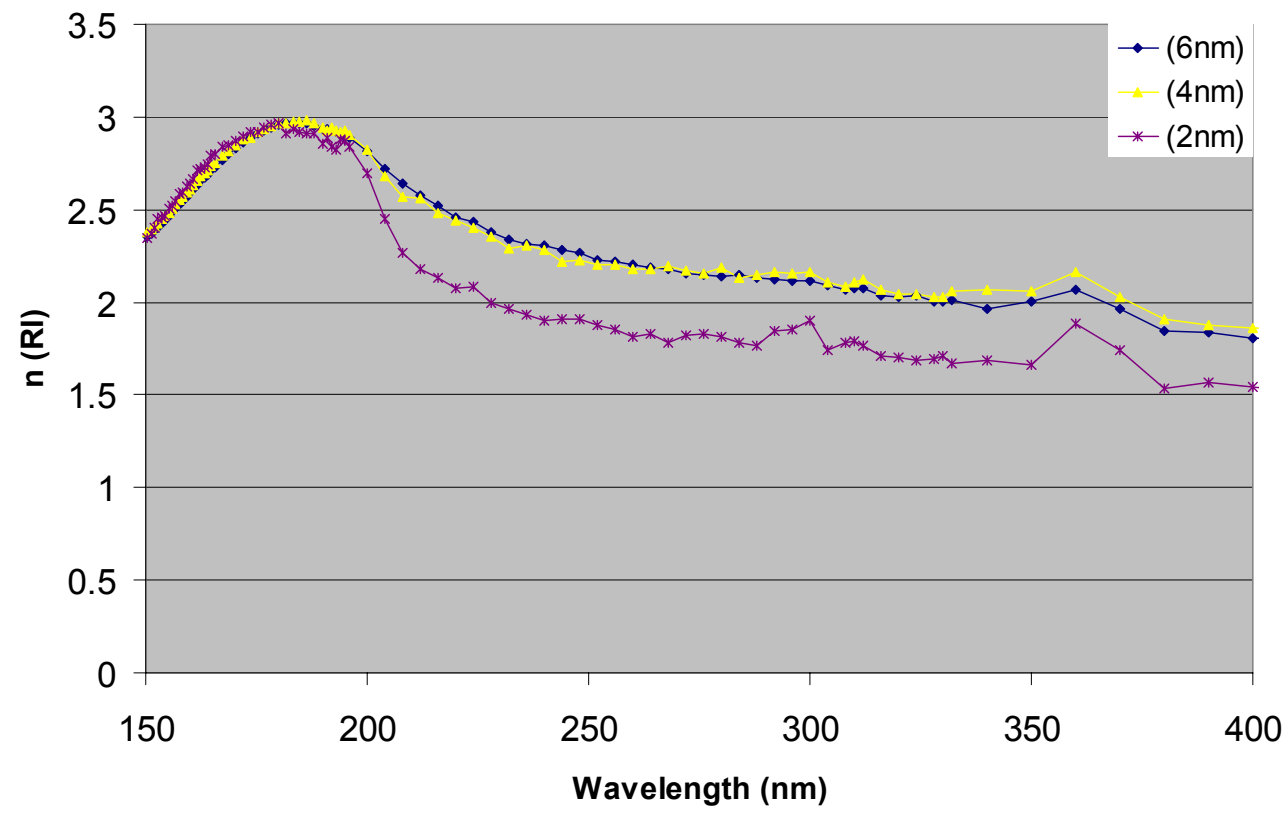

Figure 4 Thin inorganic base films of the target material for nanoparticles

The creation of non-aqueous solutions with the metal oxide nanoparticles is expected to be more of a challenge; however, terminal hydroxyls on the nanoparticle will provide a site for functionalizing the system. It has already been shown that nanoparticles can be functionalized and homogeneously dispersed in an organic medium. ${ }^{19-21}$ Care must be taken in functionalizing these nanoparticles since it is likely any addition of groups that will help disperse them in an organic fluid will also likely reduce the effective RI of the solution. 


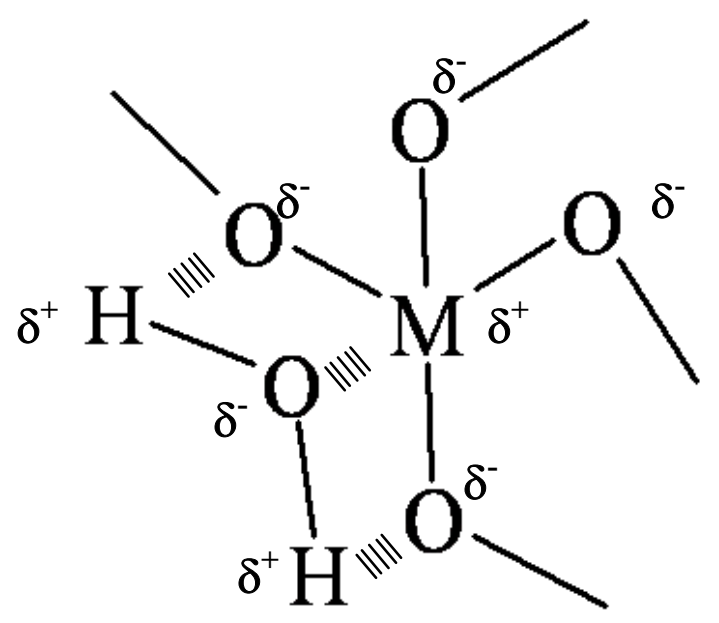

Figure 5 Schematic of the expected interaction between water and the metal oxide nanoparticles

\section{RESULTS AND DISCUSSION}

The two foremost considerations initially in this work were being able to consistently synthesize nanoparticles $<10 \mathrm{~nm}$ and being able to disperse the synthesized nanoparticles into solution. Reaction Scheme 1 shows the main reaction pathway for creating the nanoparticles. It has been determined that both reaction conditions and starting material quality are critical to obtaining the desired size and shape of nanoparticles. The basic reaction condition for producing larger metal oxide nanoparticles has been documented elsewhere. ${ }^{22}$ Figure 6 shows a transmission electron microscopy (TEM) image of the optimized nanoparticles with diameters between $2-5 \mathrm{~nm}$. If the starting materials are compromised or the reaction conditions are off slightly from the optimum, rod-shaped nanoparticles are obtained as shown in Figure 6B.

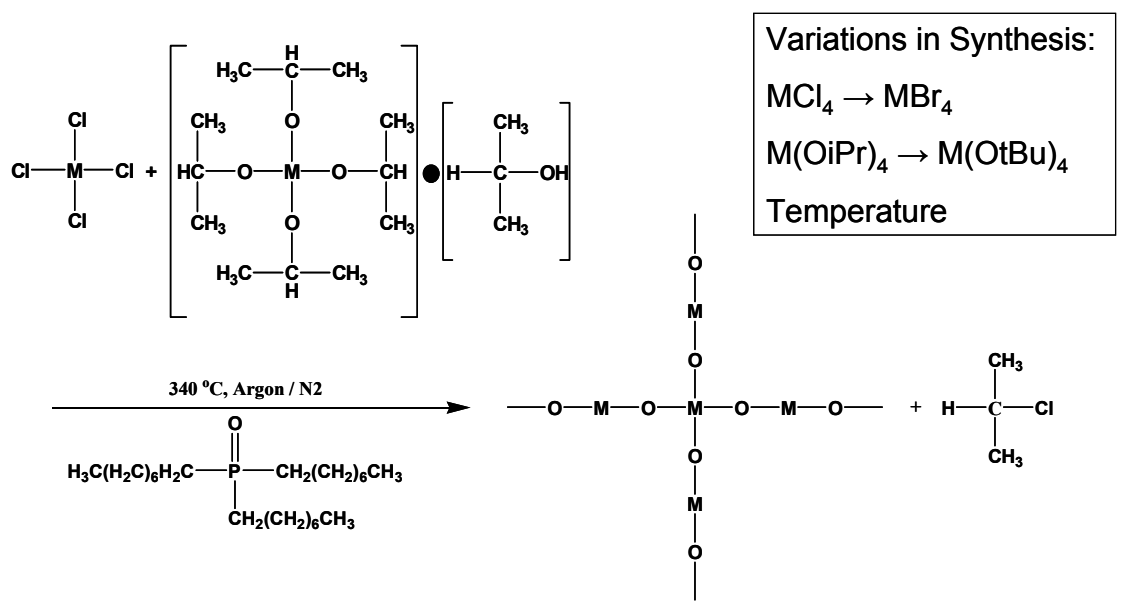

Reaction Scheme 1 General synthetic route for metal oxide nanoparticles 


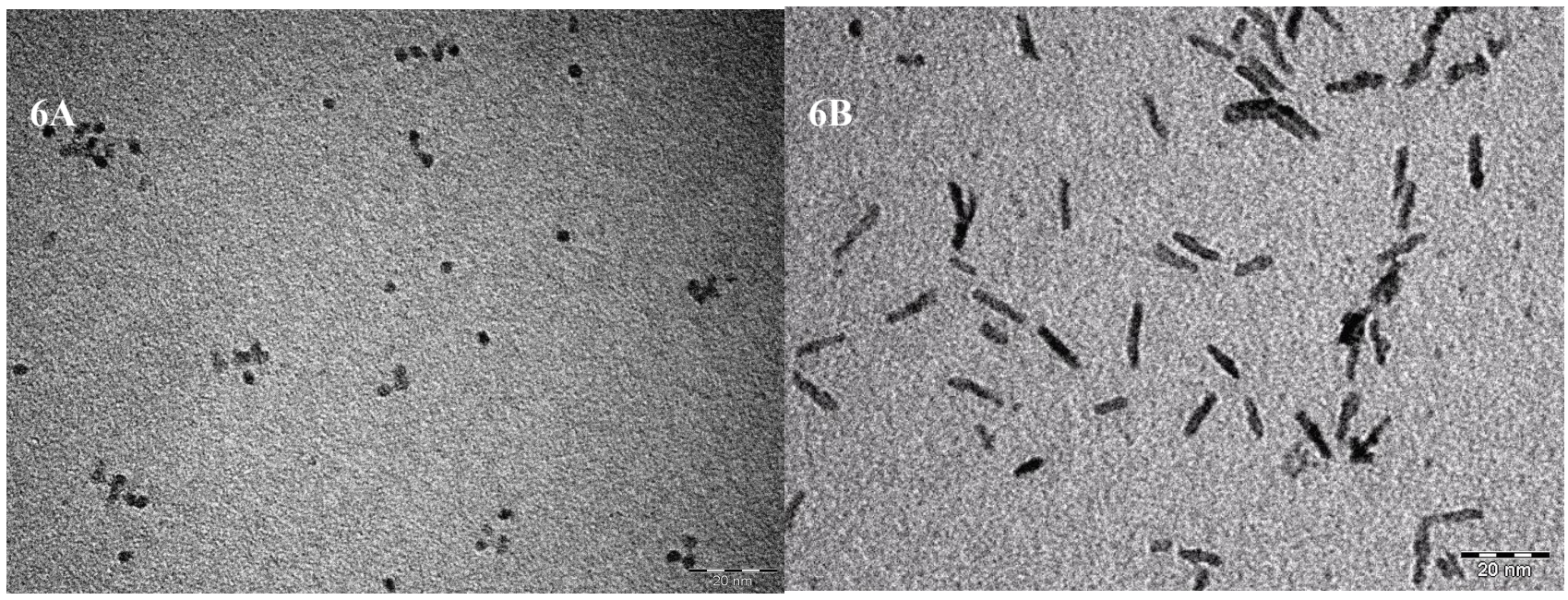

Figure 6 A) TEM showing optimized metal oxide nanoparticles obtained from the synthetic route shown above 6B) The rod-shaped nanoparticles obtained under conditions that are compromised

Initial reactions producing nanoparticles have a problem in that significant organic residue remains. Subsequent treatment with $\mathrm{H}_{2} \mathrm{O}_{2}$ and UV light reduced the amount of organic present. Figure 7 shows the Fourier transform infrared specstroscopy (FTIR) image of the nanoparticles after the standard reaction and then after treatment with $\mathrm{H}_{2} \mathrm{O}_{2}$ and UV. However, it was determined that the reaction scheme should be modified to reduce the organic content during the reaction so that a post- treatment would not be required. A second reaction scheme dubbed G2 synthesis was identified and is now the current process of record for the bulk synthesis of the nanoparticles.

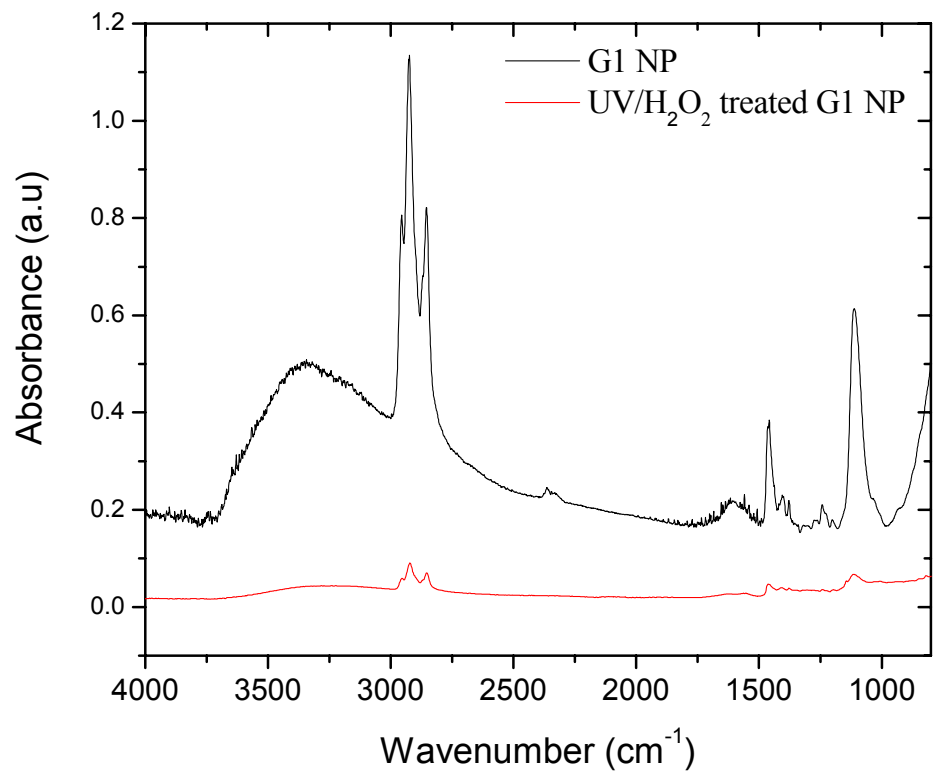

Figure 7 FTIR showing nanoparticles after the standard reaction and then after treatment with $\mathrm{H}_{2} \mathrm{O}_{2}$ and UV 
The dispersion of these nanoparticles into water was completed at various levels. Figure 8 shows the RI (@ 589nm) vs. loading for nanocomposite solutions. The solutions are shown after six days, indicating that the material does have a degree of shelf-life. It is also clear that a high loading in an aqueous medium is possible. It should be noted that the purity for this reaction scheme has not yet been optimized, resulting in a transparency that is not yet satisfactory. Loadings listed in Figure 8 are \%wt.

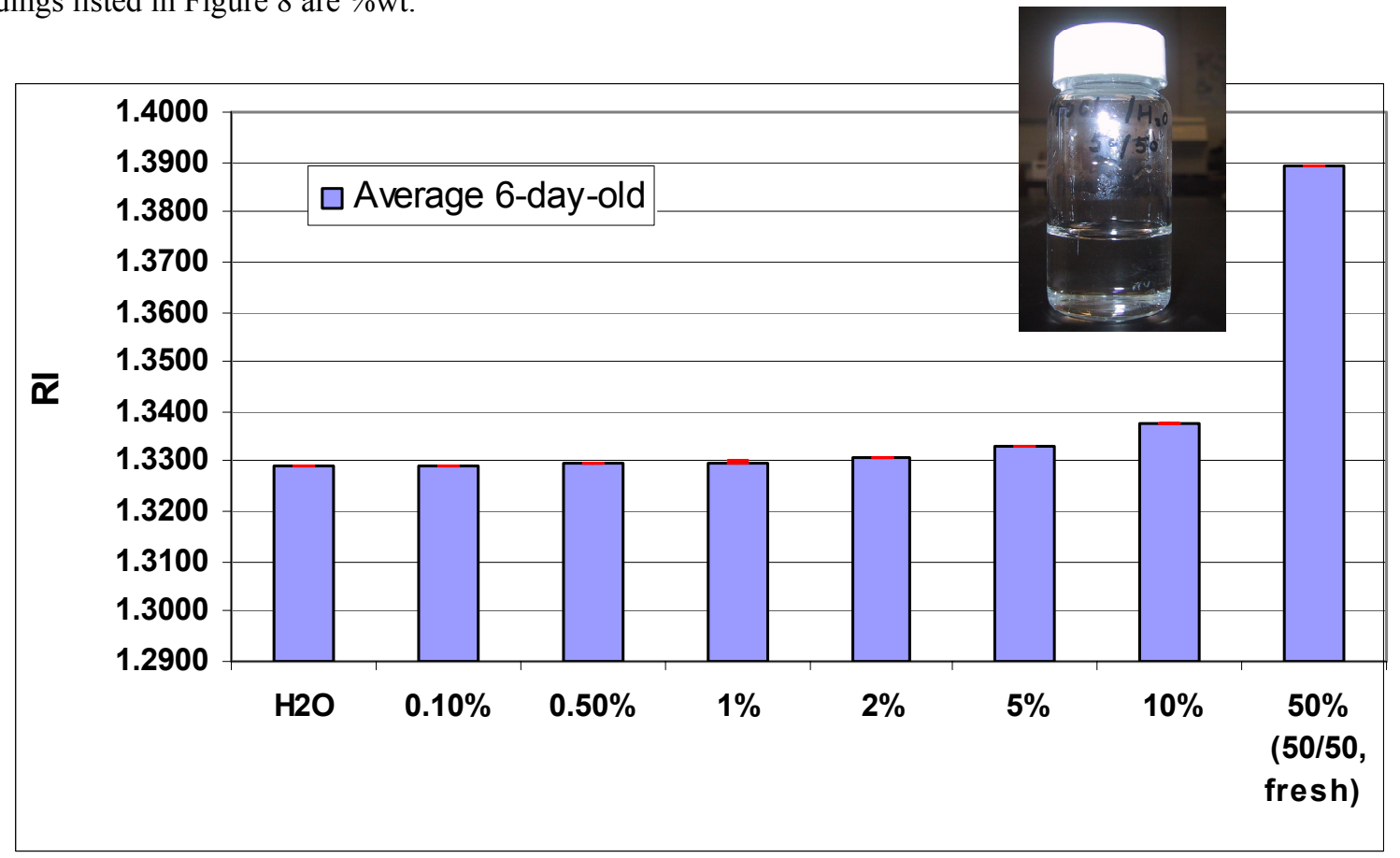

Figure 8 RI (@589nm) vs. loading for aqueous nanocomposite solutions

To obtain the refractive index values required for a Gen-2 or Gen-3, we will have to use volume \% loadings. Equation 2 is used for refractive index engineering where $\mathrm{n}_{\mathrm{f}}$ is the final desired index, $\mathrm{n}_{s}$ is the index of the solute (nanoparticle), and $\mathrm{n}_{m}$ is the index of the aqueous or organic medium. Using $\mathrm{n}_{\mathrm{f}}=1.80$ (final refractive index) and $\mathrm{n}_{\mathrm{s}}=2.9$ (transition metal oxide refractive index), we can estimate the volume fraction (Vs) of particles needed to obtain the desired refractive index. For a water-based Gen-3 fluid, a volume loading of $37 \%$ nanoparticles will be required. This may seem unrealistic when looking at a hard sphere contact model in solution; however, if we target a Gen-2 fluid at an index of 1.65 the volume loading is then $22 \%$. This is a number that may be obtainable. If, on the other hand we start with a much higher index solution such as decalin at 1.64, the volume loading becomes even more reasonable, $19 \%$, for a Gen-3 fluid.

$$
\frac{n_{f}^{2}-1}{n_{f}^{2}+2}=V_{s} \frac{n_{s}^{2}-1}{n_{s}^{2}+2}+\left(1-V_{s}\right) \frac{n_{m}^{2}-1}{n_{m}^{2}+2}
$$




\section{CONCLUSIONS}

It has been determined that the successful production of a pure organic high-index Gen-3 immersion fluid is unlikely due to fundamental limitations on how much polarizability can be introduced into organic molecules without significantly degrading their transparency. Additionally, there is a corresponding increase in reactivity for organics larger than decalin and organic fluids with heteroatoms.

The best (and perhaps the only) remaining approach for achieving a Gen-3 fluid is to use a high-index nanoparticle dispersed into solution. It has been shown that a material with an index of 2.90 at $193 \mathrm{~nm}$ can be synthesized into nanoparticles of useful dimensions. Still, much work remains in obtaining the required purity and required loadings of the nanoparticles in solutions. Further, fundamental determination of the size, which is below the scatter limit at $193 \mathrm{~nm}$, needs to be completed. Finally, interactions of the nanocomposite on the tools and surrounding materials (i.e., lens and resist) will have to be exhaustively evaluated.

\section{Disclaimers:}

Advanced Materials Research Center, AMRC, International SEMATECH Manufacturing Initiative, and ISMI are servicemarks of SEMATECH, Inc. SEMATECH, the SEMATECH logo, Advanced Technology Development Facility, ATDF, and the ATDF logo are registered servicemarks of SEMATECH, Inc. All other servicemarks and trademarks are the property of their respective owners.

\section{REFERENCES}

[1] B.W. Smith, Y. Fan, J. Zhou, A. Bourov, , L. Zavyalova, E. Piscani, J. Park, D. Summers, K. Wolf, F. Cropanese, "High index aqueous immersion fluids for 193nm and 248nm lithography", RIT Center for Nanolithography Research - Immersion fluids update, (2004) http://www.microe.rit.edu/research/lithography/research/immersion.html

[2] T. Miyamatsu, Y. Wang, M. Shima, S. Kusumoto, T. Chiba, H. Nakagawa, K. Hieda, T. Shimokawa, "Material Design for Immersion Lithography with High Refractive Index Fluid (HIF)", Advances in Resist Technology and Processing XXII. Edited by Sturtevant, John L. Proceedings of the SPIE, Volume 5753, pp. 10-19 (2005) [3] R. H. French, V. Liberman, H. V. Tran, J. Feldman, D. J. Adelman, R. C. Wheland, W. Qiu, S. J. McLain, O. Nagao, M. Kaku, M. Mocella, M. K. Yang, M. F. Lemon, L. Brubaker, A. L. Shoe, B. Fones, B. E. Fischel, K. Krohn, D. Hardy, C. Y. Chen, "High Index Immersion Lithography With Second Generation Immersion Fluids To Enable Numerical Apertures of 1.55 For Cost Effective $32 \mathrm{~nm}$ Half Pitches" Volume 6520, pp. SPIE 2007.

[4] J. Santillan, A. Otoguro, T. Itani, K. Fujii, A. Kagayama, T. Nakano, N. Nakayama, T Norio; H. Tmatani, S Fukuda, "Novel high refractive index fluids for 193nmImmersion Lithography", Optical Microlithography XIX. Edited by Flagello, Donis G. Proceedings of the SPIE, Volume 6154, pp. 1592-1599 (2006).

[5] R. H. French, H. V. Tran, D. J. Adelman, N. S. Rogado, W. Qiu1, J. Feldman, O. Nagao, M. Kaku, M. Mocella, R. C. Wheland, M. K. Yang, M. F. Lemon, L. Brubaker, B. Fones, B. E. Fischel, C. Y. Chen, "Cost Effective Single Exposure Immersion Lithography With Second Generation Immersion Fluids For Numerical Apertures of 1.55 and 32 nm Half Pitches, $4^{\text {th }}$ Intl. Symp. on Immersion Lithography, Keystone Colorado ,USA, Oct 2007.

[6] T. Furukawa, T. Kishida, T. Miyamatsu, K. Kawaguchi, K. Yamada, T. Tominaga, Z. Liu*, M. Slezak*, K. Hieda, "High Refractive Index Fluids and Top Coat Design for next generation ArF immersion lithography", $4^{\text {th }}$ Intl. Symp. on Immersion Lithography, Keystone Colorado ,USA, Oct 2007.

[7] P.A.Zimmerman, C.Van Peski, B. Rice, J. Byers, N.J.Turro, X. Lei, J.L Gejo, V. Liberman, S. Palmacci, M. Rothschild, A. Whittaker, I.Blakey, L. Chen, B. Dargaville and Heping Liu, "Status of High-Index Materials for Generation-Three 193nm Immersion Lithography, J. Poly. Sci Tech., 20(5), 643-650, 2007

[8] Laser Durability Studies of High Index Immersion Fluids: Fluid Degradation and Optics Contamination Effects , V. Liberman, M. Rothschild, S. T. Palmacci, P. A. Zimmerman, A. Grenville, , Advances in Resist Materials and Processing Technology XXIV, edited by Qinghuang LinProc. of SPIE Vol. 6519, 651921, (2007) 
[9] Outlook for potential third-generation immersion fluids, J-L Gejo, J.T. Kunjappua, J. Zhoub, B. W. Smith, P.Zimmerman, W Conley, N. J. Turro, Advances in Resist Materials and Processing Technology XXIV, edited by Qinghuang Lin Proc. of SPIE Vol. 6519, 651921, (2007)

[10] Juan Lopez-Gejo, J. T. Kunjappu, J. Zhou, B. W. Smith, P. Zimmerman, W. Conley, and N. J. Turro, Polycycloalkanes as Potential Third-Generation Immersion Fluids for Photolithography at $193 \mathrm{~nm}$, Chem. Mater. 2007, 19, 3641-3647

[11] K. Kawaguchi, T. Kishida, T. Miyamatsu, T. Tominaga, M. Slezak*, Y. Wang, K. Yamada, K. Hieda, "Third Generation Fluid for Third-ArF Immersion Lithography”, $3^{\text {rd }}$ Intl. Symp. on Immersion Lithography, Kyoto, Japan, Oct 2006.

[12] B.W. Smith, J. Zhou, F. Fan, A. Bourov, "Inorganic immersion fluids for ultrahigh numerical aperture $193 \mathrm{~nm}$ lithography," Applied Optics, Vol. 45, Issue 13, pp. 3077-3082, (2006).

[13] W. Conley, S. Warrick, C. Garza, P-J Goirand. J.-W Gemmink, D.Van Steenwinckel, "Application of Beyond Water Immersion to Device level Imaging," ${ }^{\text {rd }}$ Intl. Symp. on Immersion Lithography, Kyoto, Japan, Oct 2006.

[14] B.W. Smith, J. Zhou, "Snell or Fresnel - The influence of material index on hyper NA lithography," Proc. SPIE Optical Microlithography, In Press (2007).

[15] K.E. Peiponen and E. M Vartitianen, "Kramers-Kronig relation in Optical data inversion", Physical rev. B, 44(15), p 8301, 1991.

[16]E. Costner, K. Matsumoto, C. G. Willson, "High Index Materials for Immersion Lithography", $4^{\text {th }}$ Intl. Symp. on Immersion Lithography, Keystone Colorado ,USA, Oct 2007.

[17].S. Ando and M Ueda, "DFT calculations of photoabsorption spectrafor alicyclic and heterocyclic compounds in the VUV region", J. Polymer Sci Tech., 16(4), 537-544, 2003

[18] S. Kruger, S.Revuru, S-Z. Zhang, E. Block, P. A. .Zimmerman, D.D.Vaughn II, P. Naulleau, R.L. Brainard, "Options for High Index Fluids for Third Generation 193i Lithography", $4^{\text {th }}$ Intl. Symp. on Immersion Lithography, Keystone Colorado ,USA, Oct 2007.

19) I. Sondi, T.H. Fedynyshyn, R. Sinta, E. Matijevic, Encapsulation of Nanosized Silica by in Situ Polymerization of tert-Butyl Acrylate Monomer, Langmuir 2000, 16, 9031-9034

[20] T.H. Fedynyshyn et al., Encapsulated Inorganic Resist Technology,Proc.of SPIE Vol. 3999 (2000)

[21] T.H. Fedynyshyn et al., Encapsulated inorganic resist technology applied to $157 \mathrm{~nm}$-lithography, Proc. SPIE Vol. $4345(2001)$

[22] J. Tang, J. Fabbri, R. D. Robinson, Y. Zhu, I. P. Herman, M. L. Steigerwald, L. E. Brus, Chem. Mater., 16, 1336 (2004) 